\title{
Objective Evaluation of Facial Paralysis by Asymmetry in Expressions
}

\author{
Pujitha Gunaratne and Yukio Sato \\ Department of Electrical and Computer Engineering, \\ Nagoya Institute of Technology \\ Gokiso, Showa, Nagoya 466-8555, Japan. \\ pujitha@hilbert.elcom.nitech.ac.jp, sato@elcom.nitech.ac.jp
}

\begin{abstract}
An objective evaluation method to analyze the degree of motion dysfunction in facial expressions due to paralysis is presented. The analysis are based on range data captured for pre-determined facial actions of patients with facial paralysis. The dysfunctions in expressions are analyzed by estimating the degree of asymmetry between left and right sides of 3D expression models that were constructed from measured range data. A symmetric generic face mesh is adapted to the range data using a least squares approximation method to generate these 3D models. The 3D expression models are interpreted as inter-connected meshes of linear springs with point masses and their deformations during expressions are determined by estimating the change of energy in each case. The results obtained for patients in consecutive measurements are compared against that of normal subjects to illustrate the degree of recovery quantitatively.
\end{abstract}

\section{Introduction}

Computer assisted objective evaluation systems for the analysis of facial paralysis have been sought constantly in otorhinolaryngology. The pioneering works of Neely et al. 1 attempted to construct an objective evaluation system by capturing facial expressions using a video camera and applying image processing methods to extract the paretic motions of the face. In their work, selected black and white image frames from the recorded video clips of the expressions are analyzed against a reference image frame of the expressionless face by an image subtraction technique. These techniques are sensitive to the chromaticity of the analyzing image, which can be influenced by the change in illumination of the imaging environment and variations of skin color etc. Thus it makes a greater accountability for the robustness when expressions are captured in different time intervals, may be days or weeks, in analyzing the variations of paralysis. The method proposed by Isono et al. [2] relies on placing markers on the facial surface to track the motion during expressions. The locations of marker positions are recorded in each expression with respect to a reference point, commonly the nose tip, and their displacements are analyzed for left and right sides to determine the dysfunctions. The use of markers has its own drawbacks in repeated analysis, especially identifying similar locations for marker placement, tight binding to the skin to ensure zero relative shift during expressions etc. 
The approach proposed here successfully overcomes the drawbacks with the previous methods by implementing the analysis in 3D models constructed by range data. It neither requires the use of markers nor depends on rigid reference points for the analysis. It is also insensitive to the chromatic changes in the environment as well as the subject. In this documentation, the analysis of two facial actions, namely, the eye closure and grin actions for a patient identified with Bell's palsy are presented with similar analysis of a normal subject with no history of facial paralysis. Prior to the analysis, each facial expression is measured with a rangefinder system that produces range and color texture images of expressions in the frontal direction. For meaningful analysis of asymmetry, a well constructed generic mesh with triangular patches, which resembles face topology, is adapted to the range data of each measured expression by a least squares approximation method. The estimation of facial paralysis is done by identifying asymmetric deformations in facial surface that reflect in generated 3D mesh models. The asymmetry analysis is twofold and based on comparison of deformation in left and right side triangular patches of the 3D expression models. Firstly, deformations in patches are estimated by interpreting each mesh model as inter-connected linear springs with point masses and computing the corresponding deformation energy. Secondly, the orientation asymmetries of the patches in 3D space are analyzed. Thus, a composite asymmetry is estimated with these two factors and analyzed for patients as well as normal subjects.

\section{Construction of 3D Models}

Forced eye closure and grin expressions are measured by a rangefinder system [3], which produces 8-bit $512 \times 242$ resolution range images and $640 \times 480$ resolution color texture images in the frontal direction. This system consist of a CCD camera and two laser scanners, which are laterally mounted to measure left and right sides simultaneously. Due to this configuration, the system reports a lower measurement time, less than a second with each scanner, when measuring a human face.
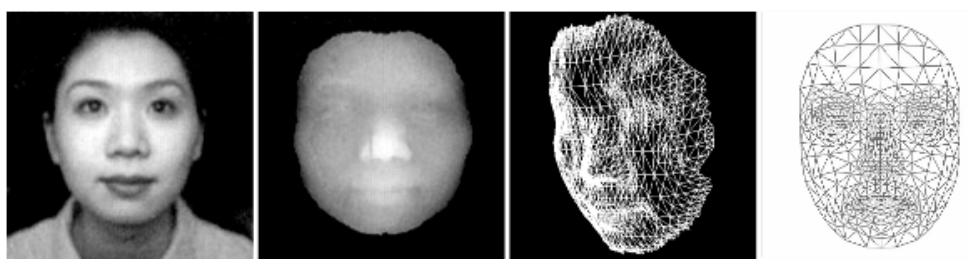

Fig. 1. Frontal texture and range images of a subject with the Adaptive mesh generated on the range data. Rightmost is the symmetric generic mesh used for adaptation.

To analyze deformation patterns in different parts of the face during expressions, the facial surface is represented with triangular patches. Although a 
straightforward approach is to apply adaptive mesh techniques to the measured range data, the meshes generated by these methods do not produce a symmetric distribution of patches in left and right sides (Fig. 1). Thus they are not suitable for the analysis. There are some attempts that have been made for automatic surface triangulation 45, which fit an adaptive canonical facial polygon network to range data automatically. However, there is no guarantee in such methods of producing identical patch densities in left and right sides of different expressions.

Therefore we adapt a pre-designed symmetric generic face mesh with triangular patches to each range image and produce 3D models of expressions that can be used in meaningful asymmetry estimations. The mesh adaptation is a tedious and time consuming process if it involves segmentation of range images to extract feature points for mapping. Instead, here we resort to a much simpler approach by extracting feature points from corresponding texture image. The range and texture images captured by this system has one-to-one correspondence, since single CCD camera with similar orientation (frontal direction) is used during image capturing. Forty-two evenly distributed mapping points in left and right sides, some of which are prominent feature points in eyes, nose and mouth regions are extracted from the frontal color image of each expression. Nose tip point is extracted simply by searching the furthermost point of the noise eliminated range image. The corresponding mesh locations of these points are pre-determined.

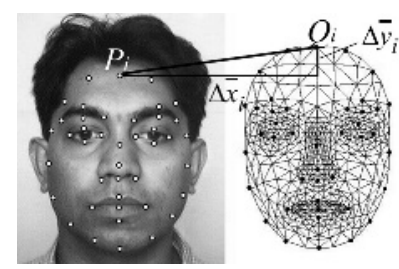

Fig. 2. Mapping points in the texture image and the face mesh with displacements along $\mathrm{X}$ and $\mathrm{Y}$ directions.

We then calculate the displacements between these feature points and corresponding mesh locations during the mapping process. Let $\Delta \overline{x_{i}}$ and $\Delta \overline{y_{i}}$ be displacement vectors along the $\mathrm{X}$ and $\mathrm{Y}$ directions of two mapping points $P_{i}$ and $Q_{i}(i=1 \ldots 42)$, on the texture image and the mesh, respectively (Fig. 2). Thus we obtain 42 displacement vectors in each direction for all mapping points. Using these points, coefficients of an $N^{t h}$ order polynomial mapping function, which is used to map remaining mesh points to the corresponding locations of the texture image by a least squares estimation, are determined.

Let $f(x, y)$ denote the mapping function given by,

$$
f(x, y)=a_{00}+\sum_{j=1}^{N} \sum_{i=0}^{j} a_{j-i, i} x^{i} y^{j-i}
$$


During initial mapping, order of the polynomial is set to $2(N=2)$. This result in a polynomial with six coefficients from $a_{00}, a_{10}, \ldots, a_{02}$. Thus with six mapping points of $P_{i}$ and $Q_{i},(i=1 \ldots 6)$, which produce six $\Delta \overline{x_{i}}$ and $\Delta \overline{y_{i}}$, the coefficients of Eq. (1), can be evaluated by substituting $x=\Delta \overline{x_{i}}$ and $y=\Delta \overline{y_{i}}$ in each case. Once the polynomial is solved, other mesh points are interpolated accordingly with the least squares approximation. The fitting error is calculated and compared with a pre-stated threshold value. If the error exceeds the threshold, we increase the order of the polynomial and repeat the same procedures of evaluating the coefficients. When $N=3,10$ coefficients and when $N=4,15$ coefficients are evaluated. Finally, depth information in the range data are mapped to the z-coordinates of the mesh points to produce a complete 3D mesh model. The details of this fitting algorithm can be found in previous publication of the authors in Ref. 6]. The altered mesh and constructed 3D models of the grin expression of a patient with Bell's palsy are depicted in Fig. 3.
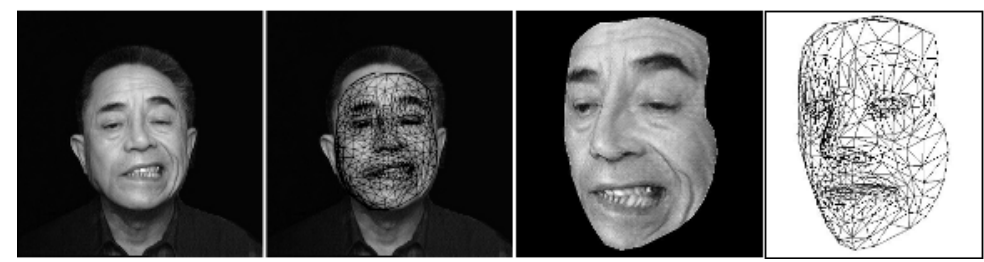

Fig. 3. The altered mesh of the grin expression and 3D models generated by applying z-values of corresponding range data.

\section{Estimation of Deformation Asymmetry}

The facial deformations during expressions are calculated based on the 3D models generated for each expression as described in the previous section. Since expressions do modify the facial surface at rest, 3D models we generated also reflect these deformations in their constituent triangular patches. To estimate these deformations, we implement 3D mesh models as meshes of connected linear springs with point masses. Suppose a particular patch in the left side consist of three springs with their gained lengths at the rest condition, from the equilibrium as $\xi_{L_{1}}, \xi_{L_{2}}$ and $\xi_{L_{3}}$ respectively (Fig. 4 ).

Thus, the energy stored in the patch at the rest condition is,

$$
E_{L_{\text {rest }}}=\frac{1}{2} k \sum_{i=1}^{3} \xi_{L} i^{2} .
$$

Where $k$ is the spring constant identical to all springs. Suppose during an expression this patch deforms to a new state with each edge modifying to lengths 


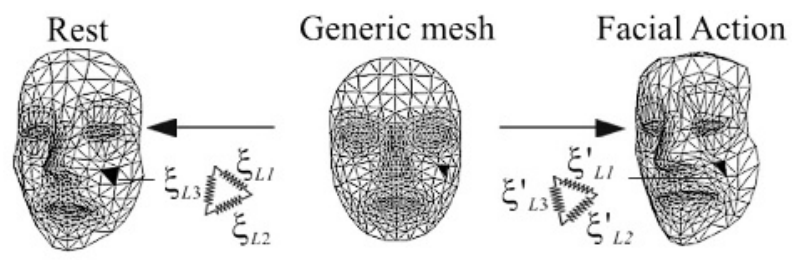

Fig. 4. Deformation of a left side patch between rest condition and an expression.

$\xi_{L_{1}}^{\prime}, \xi_{L_{2}}^{\prime}$ and $\xi_{L_{3}}^{\prime}$ respectively. Thus the change of energy from the rest condition can be stated as,

$$
\Delta E_{L}=\frac{1}{2} k \sum_{i=1}^{3}\left\|\xi_{L} i-\xi_{L}^{\prime} i\right\|^{2} .
$$

Similarly, the energy change of its mirror patch in the right side can be stated as,

$$
\Delta E_{R}=\frac{1}{2} k \sum_{i=1}^{3}\left\|\xi_{R} i-\xi_{R}^{\prime} i\right\|^{2} .
$$

Thus, if we let $\omega_{L}{ }^{2}=\sum^{3}{ }_{i=1}\left\|\xi_{L} i-\xi^{\prime}{ }_{L} i\right\|^{2}$ and $\omega_{R}{ }^{2}=\sum^{3}{ }_{i=1}\left\|\xi_{R} i-\xi_{R}^{\prime} i\right\|^{2}$ as deformations of left and right sides respectively, from Eq. (3) and Eq. (4) we can deduce $\Delta E_{L}=\frac{1}{2} k \omega_{L}^{2}$ and $\Delta E_{R}=\frac{1}{2} k \omega_{R}{ }^{2}$. Ignoring constant parts, $\omega_{L}$ and $\omega_{R}$ can be considered as candidate parameters to describe the deformation of the triangular patches.

\section{Estimation of Orientation Asymmetry}

Apart from the estimations of deformations, locally to the patches, another factor that contributes to the asymmetry is global orientation of patches in both sides even when they have identical deformations. Suppose a particular patch in the left side has the orientation $P_{L}$ in the rest condition. It changes the orientation to $P_{L}^{\prime}$ during an expression. The change in orientation during the expression can be estimated by considering the following transformations.

- Let the center of gravity of both patches $P_{L}$ and $P_{L}^{\prime}$ be $G_{L}$ and $G_{L}^{\prime}$ respectively.

- Let $N_{L}$ and $N_{L}^{\prime}$ denote the surface normal vectors of patches $P_{L}$ and $P_{L}^{\prime}$.

- Translate $G_{L}$ and $G_{L}^{\prime}$ to the origin, so that they coincide with each other.

- Align surface normal vectors $N_{L}$ and $N_{L}^{\prime}$ along the Z-axis so as to make the patches co-planer with the XY-plane.

- Calculate the direction vectors $r_{1}$ and $r_{2}$ from the center of the gravity of each patch to a similar vertex.

- Rotate the patch in XY-plane so that $r_{1}$ coincide with $r_{2}$. 


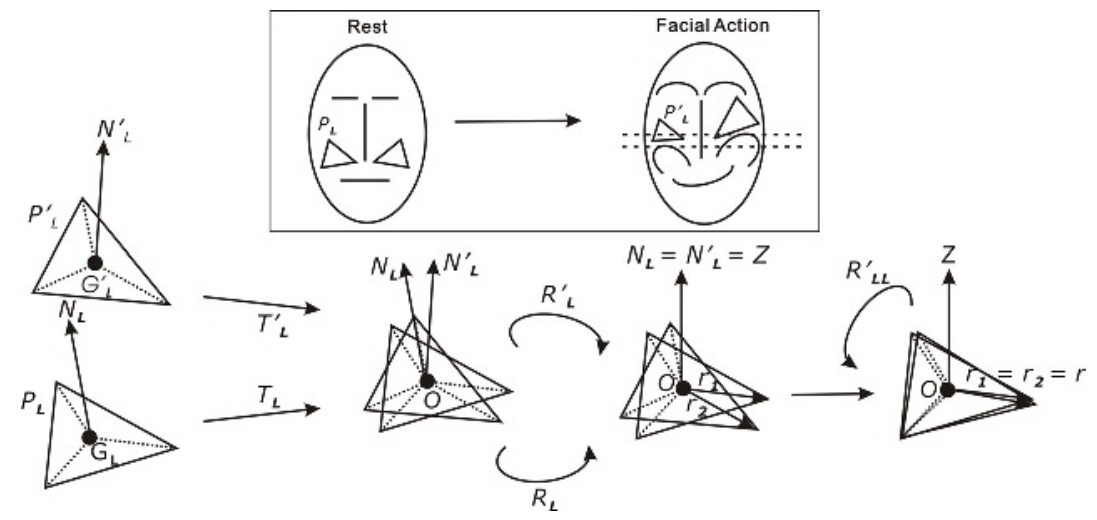

Fig. 5. Transformation of a left side patch between rest and a facial action.

This transformation scenario is depicted in Fig. 5. Thus, resulting transformation can be expressed as,

$$
T_{L} R_{L}=T_{L}^{\prime} R_{L}^{\prime} R_{L L}^{\prime \prime}
$$

We can now define the transformation parameter for the left side patches as,

$$
\sigma_{L}=T_{L} R_{L}-T_{L}^{\prime} R_{L}^{\prime} R_{L L}^{\prime \prime} .
$$

Similarly, the transformation parameter for the right side can be derived as,

$$
\sigma_{R}=T_{R} R_{R}-T_{R}^{\prime} R_{R}^{\prime} R_{R R}^{\prime \prime}
$$

Therefore the composite orientation parameter can be stated as,

$$
\sigma=\left\|\sigma_{L}-\sigma_{R}\right\|
$$

The orientation parameter can be interpreted as follows. For identical orientations of left and right side patches, $\sigma=0$. For the patches with little or no deformation during expressions (compared to the initial rest condition), $T_{L} \simeq T_{L}^{\prime}$, $T_{R} \simeq T_{R}^{\prime}, R_{L} \simeq R_{L}^{\prime}, R_{R} \simeq R_{R}^{\prime}$ and $R_{L L}^{\prime} \simeq R_{R R}^{\prime} \simeq\lceil 1\rceil$. Thus, the orientation asymmetry can be estimated for all the patches in left and right sides. Let $\eta$ be the composite asymmetry parameter given by $\eta=\omega+\sigma$. Evaluating $\eta$ for left and right side patches during expressions, a measure of asymmetric deformation can be obtained.

\section{Results}

In this work we measured eye closure and grin expressions using the rangefinder system for patients as well as normal subjects and compared the estimations.

The construction of 3D models of these expressions are described in section 2. The surface deformations are estimated on these models for each facial action 
as described in section 3. To calculate the composite deformation asymmetries, orientation of the patches in $3 \mathrm{D}$ space is evaluated as described in section 4 .
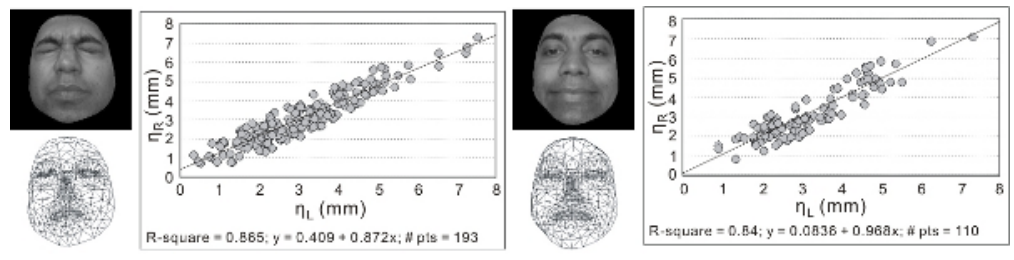

Fig. 6. Eye closure and grin actions of the normal subject.

Here we present the results of eye closure and grin actions of a patient with Bell's palsy and a normal subject with no apparent expression asymmetries. For each action, deformation asymmetries computed for corresponding sub-meshes are presented to highlight the variation patterns. A total of 193 pathes covering the eye region are considered as the eye sub-mesh, whereas, 110 patches covering mouth region are considered as mouth sub-mesh. Surface deformation and 3D orientation estimations are done for the left and right sides separately. The composite asymmetry $\eta$ is calculated for each patch in the left and right sides. For the left side patches $\eta_{L}=\omega_{L}+\sigma_{L}$ and for the right side patches $\eta_{R}=\omega_{R}+\sigma_{R}$ is evaluated. For ideally symmetric deformations, the correlation between $\eta_{L}$ and $\eta_{R}$ should confirm to a straight line of $y=m x$ type. The Fig. 6 depict the respective correlations of the eye-closure and grin actions of the normal subject.
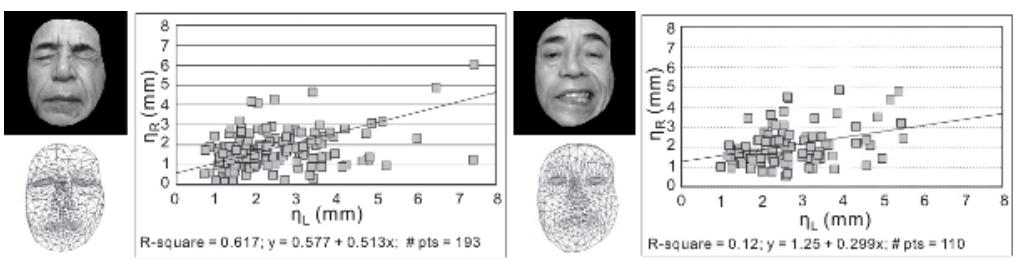

Fig. 7. Eye closure and grin actions of a patient with Bell's palsy.

Similarly, Fig. 7 depict the eye-closure and grin actions of a patient with facial paralysis. Table 1 summarize the mean and standard deviation of $\eta_{L}$ and $\eta_{R}$ of normal subject as we as the patient in eye closure and grin actions respectively.

\section{Conclusion}

In this work we have presented an approach to estimate the asymmetric deformations in facial expressions in 3D. Since the proposed method is based on 
Table 1. The mean and Standard deviations (SD) of $\eta_{L}$ and $\eta_{R}$ in eye closure and grin expressions

\begin{tabular}{lllllllll}
\hline & Eye-closure & & \multicolumn{3}{c}{ Grin } \\
& $\begin{array}{l}\text { Mean } \\
\eta_{L}\end{array}$ & & \multicolumn{3}{c}{$\begin{array}{l}\text { Std. dev. } \\
\eta_{R}\end{array}$} & $\eta_{L}$ & \multicolumn{3}{c}{ Mean } & \multicolumn{3}{c}{ Std. dev. } \\
& & & $\eta_{R}$ & $\eta_{L}$ & $\eta_{R}$ & $\eta_{L}$ & $\eta_{R}$ \\
\hline Normal sub. & 3.004 & 3.028 & 1.369 & 1.284 & 3.137 & 3.119 & 1.213 & 1.281 \\
Patient & 2.747 & 1.967 & 2.403 & 1.568 & 2.688 & 2.049 & 1.047 & 0.903 \\
\hline
\end{tabular}

the expression models made in 3D, it successfully eliminates the drawbacks of conventional methods that make use of 2D intensity images. By analyzing the correlations of asymmetry in left and right sides of the normal subject and the patient, we can confirm that the patient has paralysis in the right side in both eye closure and grin facial actions. His distributions in both expressions lean towards the $\mathrm{X}$-axis (left side) since that side produce most of the movements during expressions. It also can be noticed in the patient distributions that most of the data are concentrated on the lower regions of the graph indicating less movement during expressions compared to the normal subject. Thus proposed approach can be considered as a potential candidate in objective facial paralysis estimation environments.

Acknowledgements. We would like to thank Dr. Seiichi Nakata of Nagoya University Medical Faculty for providing patients and his keen interest in developing this system for clinical usage.

\section{References}

1. Neely, J.G., Cheung, J.Y., Wood, M., Byers, J., Rogerson, A.: Computerized quantitative dynamic analysis of facial motion in the paralyzed and synkinetic face. Am. J. Otol., Vol.13 (1992) 97-107

2. Isono, M., Murata, K., Tanaka, H., Kawamoto, M., Azuma, H.: An objective evaluation method for facial mimic motion. Otolaryngol Head Neck surg. Vol.114-1 (1996) $27-31$

3. Hasegawa, K., Hattori, K., Sato, Y.: A high speed face measurement system, Vision Interface'99 (1999) 196-202

4. Lee, Y., Terzopoulos, D., Waters, K.: Constructing physics-based facial models of individuals. In Proc. Graphics Interface '93. Canadian Information Processing Society (1993) $1-8$

5. Lee, Y., Terzoloulos, D., Waters, K.: Realistic modeling for facial animation. Computer Graphics, Vol.29(4) (1995) 55-62

6. Gunaratne, P., Sato, Y.: Three dimensional quantitative evaluation of asymmetry in facial expressions, in Proc., Sixth Digital Image Computing Techniques and Applications (DICTA’02) (2002) 7-12 